\title{
CONTINUITY OF THE HAUSDORFF MEASURE OF CONTINUED FRACTIONS \\ AND \\ COUNTABLE ALPHABET ITERATED FUNCTION SYSTEMS
}

\author{
MARIUSZ URBAŃSKI AND ANNA ZDUNIK
}

\begin{abstract}
We prove that if $J_{n}(\mathcal{G})$ we denote the set of all numbers in $[0,1]$ whose infinite continued fraction expansions have all entries in the finite set $\{1,2, \ldots, n\}$, then $\lim _{n \rightarrow \infty} \mathrm{H}_{h_{n}}\left(J_{n}(\mathcal{G})\right)=1=\mathrm{H}_{1}(J(\mathcal{G}))$, where $h_{h}$ is the Hausdorff dimension of $J_{n}(\mathcal{G})$ and $\mathrm{H}_{h_{n}}$ is the corresponding Hausdorff measure. We also show that this property is not too common by constructing a class of infinite iterated function systems $\mathcal{S}$ on $[0,1]$, consisting of similarities, for which $\varlimsup_{F \rightarrow E} \mathrm{H}_{h_{F}}\left(J_{F}\right)<\mathrm{H}_{h_{\mathcal{S}}}\left(J_{\mathcal{S}}\right)$; the upper limit is taken over finite subsets of the countable infinite alphabet $E$.
\end{abstract}

\section{INTRODUCTION}

Let $(X, \rho)$ be metric space and let $A \subset X$. Given $t \geq 0$ we define

$$
\mathrm{H}_{t}(A):=\lim _{\delta \rightarrow 0} \inf \left\{\sum_{n=1}^{\infty} \operatorname{diam}^{t}\left(U_{n}\right): \bigcup_{n=1}^{\infty} U_{n} \supset A, \operatorname{diam}\left(U_{n}\right) \leq \delta \text { for all } n \geq 1\right\}
$$

and $\mathrm{H}_{t}(A)$ is called the $t$-dimensional (outer) Hausdorff measure of $A$. The function $A \mapsto \mathrm{H}_{t}(A)$ restricted to the $\sigma$-algebra of Borel sets of $X$ is (an ordinary non-negative $\sigma$-additive) measure. The number

$$
\mathrm{HD}(A)=\inf \left\{t>0: \mathrm{H}_{t}(A)=0\right\}
$$

is called the Hausdorff dimension of $A$. Frequently, especially in dynamics, if $0<\mathrm{H}_{t}(X)<+\infty$, one considers also normalized Hausdorff measure, i.e. the function

$$
A \mapsto \mathrm{H}_{t}^{1}(A):=\mathrm{H}_{t}(A) / \mathrm{H}_{t}(X) .
$$

In order to avoid any confusion as to which Hausdorff measure we mean, we frequently refer to $\mathrm{H}_{t}(A)$ as the numerical value of the Hausdorff measure of $A$. In this paper we always consider the Hausdorff measure (and dimension) with respect to the standard (Euclidean) metric on the ambient space which is with no exception $\mathbb{R}^{q}$ with some integer $q \geq 1$.

In agreement with notation of Section 3 by $J_{n}(\mathcal{G})$ we denote the set of all numbers in $[0,1]$ whose infinite continued fraction expansions have all entries in the finite

The research of M. Urbański supported in part by the NSF Grant DMS 1001874. The research of A. Zdunik partially supported by the Polish NCN grant NN 201607940. 
set $\{1,2, \ldots, n\}$. It is well-known (see [H1], comp. [MU1] and [MU2], where an analogous statement is proved for all conformal iterated function systems), that

$$
\lim _{n \rightarrow \infty} \operatorname{HD}\left(J_{n}(\mathcal{G})\right)=1
$$

Motivated by this result and some continuity properties of the numerical value of the Hausdorff measure of the limit sets in conformal dynamics (see [Ol] and [SUZ]), we asked ourselves whether a continuity like in (1.1) holds on a deeper level of Hausdorff measures. Armed with the theory of iterated function systems it can be relatively easy to show that the continuity holds for normalized Hausdorff measures in the weak* topology on Borel probability measures on the unit interval $[0,1]$. For the numerical values of Hausdorff measures the positiver answer is given in Section 3 below; see Theorem 3.1. Its proof is in its majority number theoretical slightly touching on iterated function systems. However, this result fits well into the context of such systems. Section 4 briefly describes them and recalls Bowen's formula expressing the Hausdorff dimension of the limit set in dynamical terms. If $\mathcal{S}=\left\{\phi_{e}\right\} e \in E$ is a conformal iterated function systems satisfying the Open Set Condition, then (see [MU2]

$$
\sup \left\{\operatorname{HD}\left(J_{F}\right): F \subset E\right\}=\operatorname{HD}\left(J_{\mathcal{S}}\right)
$$

where the supremum is taken over all finite subsets $F$ of $E$ and $J_{F}$ is the limit set of the iterated function system $\left\{\phi_{e}\right\} e \in F$. Motivated by this fact and Theorem 3.1 we asked ourselves whether

$$
\lim _{F \rightarrow E} \mathrm{H}_{h_{F}}\left(J_{F}\right)=\mathrm{H}_{h_{\mathcal{S}}}\left(J_{\mathcal{S}}\right)
$$

for all conformal iterated function systems satisfying the Open Set Condition; in here $h_{F}=\operatorname{HD}\left(J_{F}\right), h_{\mathcal{S}}=\operatorname{HD}\left(J_{\mathcal{S}}\right)$ and $\mathrm{H}_{t}$ denotes always $t$-dimensional Hausdorff measure. We show in Section 6 that the answer is in general negative. It is negative already in the simplest possible situation to think about: linear (similarity), so no distortion of derivative, IFS on $[0,1]$ whose limit set is all of $[0,1]$ but a countable set (as is also the case for continued fractions). This shows that continued fractions are very special amongst IFSs on $[0,1]$. It also shows that bounded distortion of derivative, one of the main technical issues in the proof of Theorem 3.1, is by no means all what counts for the proof of this theorem. As a convenient tool to prove discontinuity in the counterexample constructed in Section 6, we derived in Section 5 a simple formula to express the Hausdorff measure of iterated function systems consisting of similarities; this formula is of interest on its own. 


\section{Selected Preliminaries from Geometric Measure Theory}

In this section we collect some well-known general density theorems which ultimately express the numerical value of Hausdorff measures in the form suitable for our continuity considerations in the following sections. We start with the following density theorem for Hausdorff measures (see [Ma] for example).

Fact 2.1. Let $X$ be a metric space, with $\mathrm{HD}(X)=h$, such that $\mathrm{H}_{h}(X)<+\infty$. Then (see p. 91 in $[\mathrm{Ma}]$ ),

$$
\lim _{r \rightarrow 0} \sup \left\{\frac{\mathrm{H}_{h}(F)}{\operatorname{diam}^{h}(F)}: x \in F, \bar{F}=F, \operatorname{diam}(F) \leq r\right\}=1
$$

for $\mathrm{H}_{h}$-a.e. $x \in X$.

As an immediate consequence of this, we get the following, fundamental for us, fact, which was extensively explored in [Ol] and [SUZ].

Theorem 2.2. If $X$ is a metric space and $0<\mathrm{H}_{h}(X)<+\infty$, then

$$
\mathrm{H}_{h}(X)=\liminf _{r \rightarrow 0}\left\{\frac{\operatorname{diam}^{h}(F)}{\mathrm{H}_{h}^{1}(F)}: x \in F, \bar{F}=F, \operatorname{diam}(F) \leq r\right\}
$$

for $\mathrm{H}_{h}^{1}$-a.e. $x \in X$.

Since in all Euclidean metric spaces the diameter of the closed convex hull of every set $A$ is the same as the diameter of $A$, as an immediate consequence of this theorem, we get the following.

Corollary 2.3. If $X$ is a subset of a Euclidean metric space $\mathbb{R}^{d}$ and $0<\mathrm{H}_{h}(X)<$ $+\infty$, then

$\mathrm{H}_{h}(X)=\liminf _{r \rightarrow 0}\left\{\frac{\operatorname{diam}^{h}(F)}{\mathrm{H}_{h}^{1}(F)}: x \in F, F \subset \mathbb{R}^{d}\right.$ is closed, convex, and $\left.\operatorname{diam}(F) \leq r\right\}$ for $\mathrm{H}_{h}^{1}-$ a.e. $x \in X$.

Being even more specific, we get the following consequence. 
Corollary 2.4. If $X$ is a subset of an interval $\Delta \subset \mathbb{R}$ and $0<\mathrm{H}_{h}(X)<+\infty$, then

$\mathrm{H}_{h}(X)=\lim _{r \rightarrow 0} \inf \left\{\frac{\operatorname{diam}^{h}(F)}{\mathrm{H}_{h}^{1}(F)}: x \in F, F \subset \Delta\right.$ is a closed interval, and $\left.\operatorname{diam}(F) \leq r\right\}$ for $\mathrm{H}_{h}^{1}$-a.e. $x \in X$.

\section{Continued Fractions}

For every integer $n \geq 1$ let $g_{n}:[0,1] \rightarrow[0,1]$ be given by the formula

$$
g_{n}(x)=\frac{1}{n+x} .
$$

Note that there exists $\xi>0$ such that for all $n \geq 1$,

$$
g_{n}\left(B_{\mathbb{C}}\left(\frac{1}{2}, \frac{1}{2}+\xi\right)\right) \subset B_{\mathbb{C}}\left(\frac{1}{2}, \frac{1}{2}+\xi\right)
$$

and $g_{n}: B_{\mathbb{C}}\left(\frac{1}{2}, \frac{1}{2}+\xi\right) \rightarrow B_{\mathbb{C}}\left(\frac{1}{2}, \frac{1}{2}+\xi\right)$ is a univalent map. The collection of maps $\mathcal{G}:=\left\{g_{n}\right\}_{n=1}^{\infty}$, acting on both $[0,1]$ and $B_{\mathbb{C}}\left(\frac{1}{2}, \frac{1}{2}+\xi\right)$, forms a conformal iterated function system in the sense of [MU2] and [MU1. It is called called the Gauss system. In view of (3.2), for every $\omega \in \mathbb{N}_{1}^{*}:=\bigcup \nu_{n \geq 1} \mathbb{N}^{n}$, say $\omega \in \mathbb{N}^{n}$, the composition

$$
g_{\omega}:=g_{\omega_{1}} \circ g_{\omega_{2}} \circ \ldots \circ g_{\omega_{n}}
$$

is a well-define self-map of both $\bar{B}_{\mathbb{C}}\left(\frac{1}{2}, \frac{1}{2}+\xi\right)$ and $[0,1]$. The map $G:(0,1] \rightarrow$ $(0,1]$, defined by the formula,

$$
G(x)=\frac{1}{x}-n \quad \text { if } \quad x \in\left(\frac{1}{n+1}, \frac{1}{n}\right],
$$

is called the Gauss map. Of course

$$
\left.G \circ g_{n}\right|_{[0,1)}=\left.\operatorname{Id}\right|_{[0,1)},
$$

and iterating this formula,

$$
G^{|\omega|} \circ g_{\omega}|[0,1)=\operatorname{Id}|_{[0,1)}
$$

for every finite word $\omega \in \mathbb{N}^{*}$, where the latter throughout this section includes the symbol " 0 " and $g_{0}:=$ Id. So, for every $k \geq 0$, and every irrational number $x \in[0,1]$,

$$
G^{k}(x) \in\left[\frac{1}{\omega(x)_{k+1}+1}, \frac{1}{\omega(x)_{k+1}}\right],
$$

where $\omega(x)_{k+1}$ is the $(k+1)$ th digit of the continued fraction expansion of $x$. Given an arbitrary non-empty subset $E$ of $\mathbb{N}$ we denote by $J_{E}(\mathcal{G})$ the set of all numbers in $[0,1]$ whose infinite continued fraction expansions have all entries in $E$. Of course

$$
g_{\omega}\left(J_{E}(\mathcal{G})\right) \subset J_{E}(\mathcal{G})
$$


CONTINUITY OF THE HAUSDORFF MEASURE OF CONTINUED FRACTIONSANDCOUNTABLE ALPHABET ITERATED F

for all $\omega \in E^{*}$, and moreover

$$
J_{E}(\mathcal{G})=\bigcup_{\omega \in E^{n}} g_{\omega}\left(J_{E}(\mathcal{G})\right)
$$

Anticipating the terminology of the next section we call $J_{E}(\mathcal{G})$ the limit set of the system $\mathcal{G}_{E}:=\left\{g_{n}: n \in E\right\}$, which is also a conformal iterated function system in the sense of [MU2] and [MU1]. In this section we exclusively consider only those systems where the set $E$ is of the form $\mathbb{N}_{n}:=\{1,2, \ldots, n\}, n \in \mathbb{N}$. We then abbreviate $\mathcal{G}_{\mathbb{N}_{n}}$ and $J_{\mathbb{N}_{n}}(\mathcal{G})$ to $\mathcal{G}_{n}$ and $J_{n}(\mathcal{G})$ respectively. Let

$$
h_{n}:=\operatorname{HD}\left(J_{n}(\mathcal{G})\right)
$$

be the Hausdorff dimension of the limit set $J_{n}(\mathcal{G})$. It follows from Theorem 1 (formula 7.11) in [H1], comp. MU2, that

$$
\lim _{n \rightarrow \infty} h_{n}=\operatorname{HD}(J(\mathcal{G}))=1,
$$

In fact Theorem 1 in [H1] provides the rate of convergence of the sequence $\left(h_{n}\right)_{n=1}^{\infty}$ to 1:

$$
\lim _{n \rightarrow \infty} n\left(1-h_{n}\right)=\frac{6}{\pi^{2}}
$$

Since each $\mathcal{G}_{n}, n \geq 2$, is a finite conformal iterated function system consisting of at least two elements, we have (see [H1] or [MU2 for instance) the following well-known result.

$$
0<\mathrm{H}_{h_{n}}\left(J_{n}(\mathcal{G})\right)<+\infty .
$$

The main result of this section is this.

\section{Theorem 3.1.}

$$
\lim _{n \rightarrow \infty} \mathrm{H}_{h_{n}}\left(J_{n}(\mathcal{G})\right)=1=\mathrm{H}_{1}(J(\mathcal{G}))
$$

Of course $\mathrm{H}_{1}(J(\mathcal{G}))=\mathrm{H}_{1}([0,1])=1$. So, only the first equality is to be proved. We start it with a long series of lemmas.

If $g: \Delta_{1} \rightarrow \Delta_{2}$ is a differentiable diffeomorphism, we define

$$
\kappa(g):=\sup \left\{\frac{\left|g^{\prime}(y)\right|}{\left|g^{\prime}(x)\right|}: x, y \in \Delta_{1}\right\}
$$

and call this number the distortion of the map $g: \Delta_{1} \rightarrow \Delta_{2}$. We say that $g$ has bounded distortion if $\kappa(g)<+\infty$. The following lemma collects the basic, straightforward to prove, properties of the concept of distortion.

Lemma 3.2. Let $\Delta_{i}, i=1,2,3$, be some three intervals in $\mathbb{R}$. Let $\operatorname{Diff}\left(\Delta_{i}, \Delta_{j}\right)$, $1 \leq i, j \leq 3$ be the set of all diffeomorphisms from $\Delta_{i}$ onto $\Delta_{j}$. Then 
(a) If $g \in \operatorname{Diff}\left(\Delta_{i}, \Delta_{j}\right)$, then $\kappa(g)=\kappa\left(g^{-1}\right)$

(b) If $g \in \operatorname{Diff}\left(\Delta_{i}, \Delta_{j}\right)$, then $\kappa(g) \geq 1$

(c) If $g_{1} \in \operatorname{Diff}\left(\Delta_{1}, \Delta_{2}\right)$ and $g_{2} \in \operatorname{Diff}\left(\Delta_{2}, \Delta_{3}\right)$, then $\kappa\left(g_{2} \circ g_{1}\right) \leq \kappa\left(g_{1}\right) \kappa\left(g_{2}\right)$.

(d) If $g \in \operatorname{Diff}\left(\Delta_{i}, \Delta_{j}\right)$ and $\Delta$ is an interval contained in $\Delta_{1}$, then

$$
\kappa^{-1}(g)\left|g^{\prime}(x)\right| \cdot|\Delta| \leq|g(\Delta)| \leq \kappa(g)\left|g^{\prime}(x)\right| \cdot|\Delta|
$$

for every $x \in \Delta_{i}$. In particular

$$
\kappa^{-1}(g) \sup \left\{\left|g^{\prime}\right|\right\} \cdot|\Delta| \leq|g(\Delta)| \leq \kappa(g) \inf \left\{\left|g^{\prime}\right|\right\} \cdot|\Delta| .
$$

It follows from (3.2) and (3.1) that

$$
g_{\omega}\left(B_{\overline{\widehat{\mathbb{C}}}}\left(\frac{1}{2}, \frac{1}{2}+\xi\right)\right) \subset B_{\overline{\widehat{\mathbb{C}}}}\left(\frac{1}{2}, \frac{1}{2}+\xi\right)
$$

for all $\omega \in \mathbb{N}^{*}$, and that all maps $g_{\omega}\left(B_{\overline{\widehat{\mathbb{C}}}}\left(\frac{1}{2}, \frac{1}{2}+\xi\right)\right) \rightarrow \mathbb{C}$ are 1-to-1 and holomorphic. As an immediate consequence of Koebe's Distortion Theorem we get therefore the following.

\section{Lemma 3.3.}

$$
\lim _{t \rightarrow 0^{+}} \sup \left\{\kappa\left(\left.g_{\omega}\right|_{\Delta}\right): \omega \in \mathbb{N}^{*} \text {, intervals } \Delta \subset[0,1] \text { with }|\Delta| \leq t\right\}=1 .
$$

Since

$$
\lim _{n \rightarrow \infty} \sup \left\{\left|g_{\omega}([0,1])\right|: \omega \in \mathbb{N}^{n}\right\}=0
$$

(the convergence is even exponentially fast), as an immediate consequence of this lemma we get the following.

\section{Lemma 3.4.}

$$
\lim _{q \rightarrow \infty} \sup \left\{\kappa\left(\left.g_{\omega}\right|_{g_{\tau}([0,1])}\right): \omega \in \mathbb{N}^{*},|\tau|=q\right\}=1 .
$$

We shall prove the following.

\section{Lemma 3.5.}

$$
\lim _{n \rightarrow \infty} \kappa\left(g_{n}\right)=1 .
$$


CONTINUITY OF THE HAUSDORFF MEASURE OF CONTINUED FRACTIONSANDCOUNTABLE ALPHABET ITERATED F

Proof. We have

$$
\left|g_{n}^{\prime}(x)\right|=\frac{1}{(x+n)^{2}}
$$

and therefore,

$$
\kappa\left(g_{n}\right)=\frac{(n+1)^{2}}{n^{2}} \rightarrow 1 \text { as } n \rightarrow \infty
$$

Since $\left|g_{n}([0,1])\right|=\frac{1}{n(n+1)} \rightarrow 0$ as $n \rightarrow \infty$, as an immediate consequence of this lemma, Lemma 3.3, and Lemma 3.2, we get the following.

\section{Lemma 3.6.}

$$
\lim _{n \rightarrow \infty} \sup \left\{\kappa\left(g_{\omega} \circ g_{n}\right): \omega \in \mathbb{N}^{*}\right\}=1
$$

We now pass to examine normalized Hausdorff measures. For every $n \geq 2$ let

$$
m_{n}:=\left.\mathrm{H}_{h_{n}}^{-1}\left(J_{n}(\mathcal{G})\right) \cdot \mathrm{H}_{h_{n}}\right|_{J_{n}(\mathcal{G})} .
$$

We also frequently consider $m_{n}$ as a Borel probability measure on [0,1], i.e.

$$
m_{n}(A)=\mathrm{H}_{h_{n}}^{-1}\left(J_{n}(\mathcal{G})\right) \cdot \mathrm{H}_{h_{n}}\left(J_{n}(\mathcal{G}) \cap A\right)
$$

for Borel subsets $A$ of $[0,1]$. It follows from [MU2] that $m_{n}$ is the unique (probability) $h_{n}$-conformal measure on $J_{n}(\mathcal{G})$, meaning that

$$
m_{n}\left(g_{\omega}(A)\right)=\int_{A}\left|g_{\omega}^{\prime}\right|^{h_{n}} d m_{n}
$$

for every Borel set $A \subset[0,1]$ and all $\omega \in \mathbb{N}_{n}^{*}$.

We start with the following definition.

Definition 3.7. A family $\mathcal{R}$ of closed subintervals of $[0,1]$ is called extremal if

$$
\liminf _{n \rightarrow \infty} \inf \left\{\frac{|\Delta|^{h_{n}}}{m_{n}(\Delta)}: \Delta \in \mathcal{R}\right\} \geq 1 .
$$

Lemma 3.8. For every $\delta>0$ the family $\mathcal{R}_{\delta}$ of all closed intervals $\Delta \subset[0,1]$ with $|\Delta| \geq \delta$ is extremal. 
Proof. Suppose on the contrary that for some $\delta>0$ the family $\mathcal{R}_{\delta}$ is not extremal. This means that there exist $\eta \in[0,1)$, an increasing sequence $\left(n_{j}\right)_{1}^{\infty}$ of positive integers, and a sequence $\left(\Delta_{j}\right)_{1}^{\infty}$ of closed intervals in $\mathcal{R}_{\delta}$ such that

$$
\lim _{j \rightarrow \infty} \frac{\left|\Delta_{j}\right|^{h_{n_{j}}}}{m_{n_{j}}\left(\Delta_{j}\right)}=\eta \text {. }
$$

Passing to a subsequence we may assume that the left-hand endpoints and the right-hand endpoints of $\Delta_{j}$ converge respectively to $a$ and $b$ in $[0,1]$ with $b-a \geq \delta$.

Let $\Delta:=[a, b] \in \mathcal{R}_{\delta}$. Since the sequence $\left(m_{n_{j}}\right)_{1}^{\infty}$ converges weakly to $m$, the Lebesgue measure on $[0,1]$, we get from (3.6) that

$$
1=\frac{|\Delta|}{m(\Delta)} \leq \frac{\lim _{j \rightarrow \infty}\left|\Delta_{j}\right|^{h_{n_{j}}}}{\limsup _{j \rightarrow \infty} m_{n_{j}}\left(\Delta_{j}\right)}=\liminf _{j \rightarrow \infty} \frac{\left|\Delta_{j}\right|^{h_{n_{j}}}}{m_{n_{j}}\left(\Delta_{j}\right)}=\eta<1 .
$$

This contradiction finishes the proof.

\section{Lemma 3.9.}

$$
\liminf _{\substack{n \rightarrow \infty \\ r \rightarrow 0}}\left\{\frac{r^{h_{n}}}{m_{n}([0, r])}\right\} \geq 1 .
$$

Proof. Fix $N \geq 2$ so large that $h_{N} \geq 3 / 4$ and keep always $n \geq N$. For every $\in(0,1 / 2)$ let $s_{r} \geq 1$ be the unique integer such that

$$
\frac{1}{s_{r}+1}<r \leq \frac{1}{s_{r}} \text {. }
$$

We then have

$$
\begin{aligned}
m_{n}([0, r]) & \leq \sum_{j=s_{r}}^{\infty} m_{n}\left(g_{j}([0,1])\right) \leq \sum_{j=s_{r}}^{\infty}\left\|g_{j}^{\prime}\right\|_{\infty}^{h_{n}} m_{n}([0,1]) \\
& \leq \sum_{j=s_{r}}^{\infty} j^{-2 h_{n}} \\
& \leq \int_{s_{r}-1}^{\infty} x^{-2 h_{n}} d x \\
& =\left(2 h_{n}-1\right)^{-1}\left(s_{r}-1\right)^{1-2 h_{n}} .
\end{aligned}
$$

Therefore,

$$
\begin{aligned}
\frac{m_{n}([0, r])}{r^{h_{n}}} & \leq\left(2 h_{n}-1\right)^{-1}\left(s_{r}+1\right)^{h_{n}}\left(s_{r}-1\right)^{1-2 h_{n}} \\
& =\left(2 h_{n}-1\right)^{-1}\left(\frac{s_{r}+1}{s_{r}-1}\right)^{h_{n}}\left(s_{r}-1\right)^{1-h_{n}} \\
& =\left(2 h_{n}-1\right)^{-1}\left(1+\frac{2}{s_{r}-1}\right)^{h_{n}}\left(s_{r}-1\right)^{1-h_{n}} \\
& \leq\left(2 h_{n}-1\right)^{-1}(1+4 r)^{h_{n}}\left(s_{r}-1\right)^{1-h_{n}}
\end{aligned}
$$


CONTINUITY OF THE HAUSDORFF MEASURE OF CONTINUED FRACTIONSANDCOUNTABLE ALPHABET ITERATED F

Of course if $0<r \leq \frac{1}{n+1}$, then

$$
\frac{m_{n}([0, r])}{r^{h_{n}}}=0 \text {. }
$$

Hence, we can continue (3.7) assuming that $r>\frac{1}{n+1}$. Then $s_{r}<n+1$, and (3.7) along with 3.4 yield for all $n \geq 2$ large enough the following.

$$
\begin{aligned}
\frac{m_{n}([0, r])}{r^{h_{n}}} & \leq\left(2 h_{n}-1\right)^{-1}(1+4 r) n^{1-h_{n}} \leq\left(2 h_{n}-1\right)^{-1}(1+4 r) n^{\frac{7}{\pi^{2} n}} \\
& =\left(2 h_{n}-1\right)^{-1}\left(n^{\frac{1}{n}}\right)^{\frac{7}{\pi^{2}}}(1+4 r) .
\end{aligned}
$$

Since $\lim _{n \rightarrow \infty} h_{n}=1$ and $\lim _{n \rightarrow \infty} n^{\frac{1}{n}}=1$, this formula gives

$$
\limsup _{\substack{n \rightarrow \infty \\ r \rightarrow 0}}\left\{\frac{m_{n}([0, r])}{r^{h_{n}}}\right\} \leq 1 \text {. }
$$

The proof is complete.

For every $\omega \in \mathbb{N}^{*}$ let

$$
B_{*}\left(g_{\omega}(0), r\right)=\left[g_{\omega}(0), g_{\omega}(0)+r\right] \text { and } B_{*}\left(g_{\omega}(0), r\right)=\left[g_{\omega}(0)-r, g_{\omega}(0)\right]
$$

respectively if $|\omega|$ is even or odd. Let

$$
B_{*}(\omega):=\left\{B_{*}\left(g_{\omega}(0), r\right): r \in(0,1]\right\}
$$

We shall prove the following.

Lemma 3.10. For every $\omega \in \mathbb{N}^{*}$ the family $B_{*}(\omega)$ is extremal.

Proof. For all $r \in\left(0,\left|g_{\omega}([0,1])\right|\right]$ there exists a unique $\hat{r} \in(0,1]$ such that

$$
B_{*}\left(g_{\omega}(0), r\right)=g_{\omega}([0, \hat{r}]) .
$$

By virtue of Lemma $3.2(\mathrm{~d})$ this gives,

$$
\kappa^{-1}\left(\left.g_{\omega}\right|_{[0, \hat{r}]}\right)\left|g_{\omega}^{\prime}(0)\right| \hat{r} \leq r \leq \kappa\left(\left.g_{\omega}\right|_{[0, \hat{r}]}\right)\left|g_{\omega}^{\prime}(0)\right| \hat{r} .
$$

Hence,

$$
\begin{aligned}
\frac{r^{h_{n}}}{m_{n}\left(B_{*}\left(g_{\omega}(0), r\right)\right)} & \geq \frac{\kappa^{-h_{n}}\left(\left.g_{\omega}\right|_{[0, \hat{r}]}\right)\left|g_{\omega}^{\prime}(0)\right|^{h_{n}} \hat{r}^{h_{n}}}{\kappa^{h_{n}}\left(\left.g_{\omega}\right|_{[0, \hat{r}]}\right)\left|g_{\omega}^{\prime}(0)\right|^{h_{n}} m_{n}([0, r])} \\
& =\kappa^{-2 h_{n}}\left(\left.g_{\omega}\right|_{[0, \hat{r}]}\right) \frac{\hat{r}^{h_{n}}}{m_{n}([0, r])} .
\end{aligned}
$$

Since $\lim _{r \rightarrow 0} \hat{r}=0$, as an immediate consequence of Lemma 3.9 and Lemma 3.3 . we get the following.

$$
\liminf _{\substack{n \rightarrow \infty \\ r \rightarrow 0}} \frac{r^{h_{n}}}{m_{n}\left(B_{*}\left(g_{\omega}(0), r\right)\right)} \geq 1
$$


This means that for every $\varepsilon>0$ there exist an integer $N_{\varepsilon} \geq 2$ and a radius $R_{\varepsilon} \in(0,1]$ such that

$$
\frac{r^{h_{n}}}{m_{n}\left(B_{*}\left(g_{\omega}(0), r\right)\right)} \geq 1-\varepsilon
$$

for all $n \geq N_{\varepsilon}$ and all $0<r \leq R_{\varepsilon}$. Invoking now Lemma 3.8, we therefore get

$$
\begin{aligned}
\varliminf_{n \rightarrow \infty} & \inf _{r \in(0,1]}\left\{\frac{r^{h_{n}}}{m_{n}\left(B_{*}\left(g_{\omega}(0), r\right)\right)}\right\}= \\
& =\varliminf_{n \rightarrow \infty} \min \left\{\inf _{r \in\left(0, R_{\varepsilon}\right]}\left\{\frac{r^{h_{n}}}{m_{n}\left(B_{*}\left(g_{\omega}(0), r\right)\right)}\right\}, \inf _{r \in\left(R_{\varepsilon}, 1\right]}\left\{\frac{r^{h_{n}}}{m_{n}\left(B_{*}\left(g_{\omega}(0), r\right)\right)}\right\}\right\} \\
& \geq \min \left\{\varliminf_{n \rightarrow \infty} \inf _{r \in\left(0, R_{\varepsilon}\right.}\left\{\frac{r^{h_{n}}}{m_{n}\left(B_{*}\left(g_{\omega}(0), r\right)\right)}\right\}, \varliminf_{n \rightarrow \infty} \inf _{r \in\left(R_{\varepsilon}, 1\right]}\left\{\frac{r^{h_{n}}}{m_{n}\left(B_{*}\left(g_{\omega}(0), r\right)\right)}\right\}\right\} \\
& \geq \min \{1-\varepsilon, 1\}=1-\varepsilon .
\end{aligned}
$$

Letting now $\varepsilon \rightarrow 0^{+}$our lemma follows.

Now consider an arbitrary finite word $\omega \in \mathbb{N}^{*}$. Put $k=|\omega|$. Since

$$
g_{\omega}(0)=g_{\left.\omega\right|_{k-1}\left(\omega_{k}-1\right) 1}(0)
$$

if $\omega_{k} \geq 2$, and

$$
g_{\omega}(0)=g_{\left.\omega\right|_{k-2}\left(\omega_{k-1}+1\right)}(0)
$$

if $\omega_{k}=1$, and since $|\omega|_{k-1}\left(\omega_{k}-1\right) 1|=| \omega \mid+1$ and $|\omega|_{k-2}\left(\omega_{k-1}+1\right)|=| \omega \mid-1$, as an immediate consequence of Lemma 3.10 , we get the following.

Corollary 3.11. For every $\omega \in \mathbb{N}^{*}$ let $\mathcal{R}_{e}(\omega)$ be the collection of all closed intervals $\Delta$ in $[0,1]$ having $g_{\omega}(0)$ as one of its endpoints. Then each family $\mathcal{R}_{e}(\omega)$, $\omega \in \mathbb{N}^{*}$, is extremal.

If $\mathcal{R}$ and $\mathcal{S}$ are two families of closed subintervals of $[0,1]$, then

$$
\mathcal{R} * \mathcal{S}:=\{\Delta \cup \Gamma: \Delta \in \mathcal{R}, \Gamma \in \mathcal{S} \text {, and } \#(\Delta \cap \Gamma)=1\} .
$$

Of course the operation "*" is is associative and commutative. Generalizing Definition 3.7 we introduce the following.

Definition 3.12. A sequence $\left(\mathcal{R}_{k}\right)_{1}^{\infty}$ of families of closed subintervals of $[0,1]$ is called extremal if

$$
\liminf _{\substack{n \rightarrow \infty \\ k \rightarrow \infty}} \inf \left\{\frac{|\Delta|^{h_{n}}}{m_{n}(\Delta)}: \Delta \in \mathcal{R}_{k}\right\} \geq 1
$$


CONTINUITY OF THE HAUSDORFF MEASURE OF CONTINUED FRACTIONSANDCOUNTABLE ALPHABET ITERATED F

The first obvious observations are these.

Lemma 3.13. If for every $k \geq 1, \mathcal{R}_{k} \subset \mathcal{S}_{k}$ and the sequence $\left(\mathcal{S}_{k}\right)_{1}^{\infty}$ is extremal, then the sequence $\left(\mathcal{R}_{k}\right)_{1}^{\infty}$ is also extremal.

Lemma 3.14. A sequence $\left(\mathcal{R}_{k}\right)_{1}^{\infty}$ is extremal if and only if the sequence $\left(\bigcup_{l=k} \mathcal{R}_{l}\right)_{k=1}^{\infty}$ is extremal.

Lemma 3.15. If $\left(\mathcal{R}_{k}\right)_{1}^{\infty}$ and $\left(\mathcal{S}_{k}\right)_{1}^{\infty}$ are two extremal sequences, then the sequence $\left(\mathcal{R}_{k} \cup \mathcal{S}_{k}\right)_{1}^{\infty}$ is also extremal.

Now we shall prove the following slightly more involved lemma.

Lemma 3.16. If $\left(\mathcal{R}_{k}\right)_{1}^{\infty}$ and $\left(\mathcal{S}_{k}\right)_{1}^{\infty}$ are two extremal sequences, then the sequence $\left(\mathcal{R}_{k} * \mathcal{S}_{k}\right)_{1}^{\infty}$ is also extremal.

Proof. Fix $\varepsilon>0$. By our hypothesis there exists $N_{\varepsilon} \geq 2$ such that

$$
\frac{|\Gamma|^{h_{n}}}{m_{n}(\Gamma)} \geq 1-\varepsilon
$$

for all $n, k \geq N_{\varepsilon}$ and all $\Gamma \in \mathcal{R}_{k} \cup \mathcal{S}_{k}$. Fix $n, k \geq N_{\varepsilon}$ and $\Delta \in \mathcal{R}_{k} * \mathcal{S}_{k}$. This means that

$$
\Delta=\Delta_{-} \cup \Delta_{+}
$$

with some $\Delta_{-} \in \mathcal{R}_{k}$ and $\Delta_{+} \in \mathcal{S}_{k}$ such that $\mathbb{D}_{-} \cap \Delta_{+}$is a singleton. Now the standard calculus argument shows that

$$
x^{t}+(1-x)^{t} \leq 2^{1-t}
$$

for all $t, x \in[0,1]$. Therefore we get

$$
\frac{\left|\Delta_{-}\right|^{h_{n}}+\left|\Delta_{+}\right|^{h_{n}}}{\left(\left|\Delta_{-}\right|+\left|\Delta_{+}\right|\right)^{h_{n}}}=\left(\frac{\left|\Delta_{-}\right|}{\left|\Delta_{-}\right|+\left|\Delta_{+}\right|}\right)^{h_{n}}+\left(\frac{\left|\Delta_{-}\right|}{\left|\Delta_{+}\right|+\left|\Delta_{+}\right|}\right)^{h_{n}} \leq 2^{1-h_{n}} .
$$


Hence, using also (3.9), we get

$$
\begin{aligned}
\frac{|\Delta|^{h_{n}}}{m_{n}(\Delta)} & =\frac{\left(\left|\Delta_{-}\right|+\left|\Delta_{+}\right|\right)^{h_{n}}}{m_{n}\left(\Delta_{-}\right)+m_{n}\left(\Delta_{+}\right)} \\
& \geq 2^{h_{n}-1} \frac{\left|\Delta_{-}\right|^{h_{n}}+\left|\Delta_{+}\right|^{h_{n}}}{m_{n}\left(\Delta_{-}\right)+m_{n}\left(\Delta_{+}\right)} \\
& \geq 2^{h_{n}-1} \min \left\{\frac{\left|\Delta_{-}\right|^{h_{n}}}{m_{n}\left(\Delta_{-}\right)}, \frac{\left|\Delta_{+}\right|^{h_{n}}}{m_{n}\left(\Delta_{+}\right)}\right\} \\
& \geq(1-\varepsilon) 2^{h_{n}-1}
\end{aligned}
$$

Invoking (3.3) this completes the proof.

If $\mathcal{R}$ and $\mathcal{S}$ are two families of closed subintervals of [0,1], we define

$$
\mathcal{R} \otimes \mathcal{S}:=\mathcal{R} \cup(\mathcal{R} * \mathcal{S}) \cup \mathcal{S}
$$

As an immediate consequence of Lemma 3.15 and Lemma 3.16 we get the following.

Corollary 3.17. If $\left(\mathcal{R}_{k}\right)_{1}^{\infty}$ and $\left(\mathcal{S}_{k}\right)_{1}^{\infty}$ are two extremal sequences, then the sequence $\left(\mathcal{R}_{k} \otimes \mathcal{S}_{k}\right)_{1}^{\infty}$ is also extremal.

An immediate induction then yields the following.

Lemma 3.18. If $T$ is a finite set and for every $t \in T$ a sequence $\left(\mathcal{R}_{k}(t)\right)_{k=1}^{\infty}$ is extremal, then the sequence $\left(\otimes_{t \in T} \mathcal{R}_{k}(t)\right)_{k=1}^{\infty}$ is also extremal.

Applying this lemma to a constant sequence we get the following.

Corollary 3.19. If $T$ is a finite set and for every $t \in T$ a family $\mathcal{R}(t)$ is extremal, then the family $\otimes_{t \in T} \mathcal{R}(t)$ is also extremal.

For every $\omega \in \mathbb{N}^{*}$ let $\mathcal{R}(\omega)$ be the collection of all closed intervals $\Delta$ in $[0,1]$ containing $g_{\omega}(0)$. We can now easily upgrade Corollary 3.11 to the following.

Lemma 3.20. For every $\omega \in \mathbb{N}^{*}$ family $\mathcal{R}(\omega), \omega \in \mathbb{N}^{*}$, is extremal.

Proof. It suffices to notice that $\mathcal{R}(\omega)=\mathcal{R}_{e}(\omega) \otimes \mathcal{R}_{e}(\omega)$ and to apply Corollary 3.11 along with Lemma 3.18 . 
Now for every integer $k \geq 1$ let $\mathcal{S}_{k}^{-}$be the family of all intervals of the form

$$
\left[\frac{1}{k}-r, \frac{1}{k}\right], r \in\left[0, \frac{1}{k(k+1)}\right] \text {. }
$$

We shall prove the following.

Lemma 3.21. The sequence $\left(\mathcal{S}_{k}^{-}\right)_{1}^{\infty}$ is extremal.

Proof. We start the proof in the same way as the proof of Lemma 3.10 with $\omega=k$.

Formula (3.8) then says that

$$
\frac{r^{h_{n}}}{m_{n}\left(\left[\frac{1}{k}-r, \frac{1}{k}\right]\right)} \geq \kappa^{-2}\left(\left.g_{k}\right|_{0, \hat{r}]}\right) \frac{\hat{r}^{h_{n}}}{m_{n}([0, \hat{r}])} \geq \kappa^{-2}\left(g_{k}\right) \frac{\hat{r}^{h_{n}}}{m_{n}([0, \hat{r}])}
$$

for all $r \in\left[0, \frac{1}{k(k+1)}\right]$. Invoking now Lemma 3.10 and Lemma 3.5 completes the proof.

Now for every integer $k \geq 1$ let $\mathcal{S}_{k}^{+}$be the family of all intervals of the form

$$
\left[\frac{1}{k+1}, \frac{1}{k+1}+r\right], r \in\left[0, \frac{1}{k(k+1)}\right] \text {. }
$$

We shall prove the following.

Lemma 3.22. The sequence $\left(\mathcal{S}_{k}^{+}\right)_{1}^{\infty}$ is extremal.

Proof. Observe that for every $r \in\left[0, \frac{1}{k(k+1)}\right]$ there exists a unique $\tilde{r} \in[0,1]$ such that

$$
\left[\frac{1}{k+1}, \frac{1}{k+1}+r\right]=g_{k}([1-\tilde{r}, 1]) \text {. }
$$

Proceeding now in the same way as that leading to (3.8), we get the following.

$$
\frac{r^{h_{n}}}{m_{n}\left(\left[\frac{1}{k+1}, \frac{1}{k+1}+r\right]\right)} \geq \kappa^{-2}\left(\left.g_{k}\right|_{[1-\tilde{r}, 1]}\right) \frac{\tilde{r}^{h_{n}}}{m_{n}([1,1-\tilde{r}])} \geq \kappa^{-2}\left(g_{k}\right) \frac{\tilde{r}^{h_{n}}}{m_{n}([1,1-\tilde{r}])} .
$$

Invoking now Lemma 3.5 and Corollary 3.11 (with $\omega=1$ ), completes the proof.

Now we shall prove a purely computational lemma.

\section{Lemma 3.23.}

$$
\varlimsup_{k \rightarrow \infty} \sup \left\{\frac{(k-1)^{-a}-(k-1+q)^{-a}}{k^{-a}-(k+q)^{-a}}: \alpha \in[1 / 2,1], q \geq 1\right\} \leq 1 .
$$


Proof. We have for all $\alpha \in[1 / 2,1]$ all $q \geq 1$, and all $k \geq 2$ that

$$
\begin{aligned}
\frac{(k-1)^{-a}-(k-1+q)^{-a}}{k^{-a}-(k+q)^{-a}} & =\left(\frac{k-1}{k}\right)^{-\alpha} \frac{\left(1-\left(\frac{k-1+q}{k-1}\right)^{-\alpha}\right)}{\left(1-\left(\frac{k+q}{k}\right)^{-\alpha}\right)} \\
& =\left(\frac{k}{k-1}\right)^{\alpha} \frac{\left(1-\left(1+\frac{q}{k-1}\right)^{-\alpha}\right)}{\left(1-\left(1+\frac{q}{k}\right)^{-\alpha}\right)} \\
& \leq\left(\frac{k}{k-1}\right) \frac{1-\left(1+\frac{q}{k-1}\right)^{-\alpha}}{1-\left(1+\frac{q}{k}\right)^{-\alpha}} .
\end{aligned}
$$

Since $\lim _{k \rightarrow \infty} \frac{k}{k-1}=1$, it is therefore enough to show that

$$
\varlimsup_{k \rightarrow \infty} \sup \left\{\frac{1-\left(1+\frac{q}{k-1}\right)^{-\alpha}}{1-\left(1+\frac{q}{k}\right)^{-\alpha}}: \alpha \in[1 / 2,1], q \geq 1\right\} \leq 1 .
$$

With $\alpha \in[1 / 2,1]$ let

$$
\psi_{\alpha}(t)=1-(1+t)^{-\alpha}, \quad t \geq 0 .
$$

The Mean Value Theorem then gives.

$$
\begin{aligned}
\psi_{\alpha}\left(\frac{q}{k-1}\right)-\psi_{\alpha}\left(\frac{q}{k}\right) & =\alpha\left(\frac{q}{k-1}-\frac{q}{k}\right)(1+\xi)^{-(1+\alpha)} \leq \frac{q}{k(k-1)}(1+\xi)^{-(1+\alpha)} \\
& \leq \frac{2 q}{k^{2}}(1+\xi)^{-(1+\alpha)} \\
& \leq \frac{2 q}{k^{2}}\left(1+\frac{q}{k}\right)^{-(1+\alpha)}
\end{aligned}
$$

for some $\xi \in\left[\frac{q}{k}, \frac{q}{k-1}\right]$ and all $k \geq 2$. Now, if $q \geq k$, then $\psi_{\alpha}(q / k) \geq 1-2^{-\alpha} \geq$ $1-2^{-\frac{1}{2}}=1-\frac{\sqrt{2}}{2}>0$. Hence,

$$
\begin{aligned}
\frac{\psi_{\alpha}\left(\frac{q}{k-1}\right)-\psi_{\alpha}\left(\frac{q}{k}\right)}{\psi_{\alpha}\left(\frac{q}{k}\right)} & \leq 2\left(1-\frac{\sqrt{2}}{2}\right)^{-1} \frac{q}{k^{2}}\left(\frac{q}{k}\right)^{-(1+\alpha)}=2\left(1-\frac{\sqrt{2}}{2}\right)^{-1} q^{-\alpha} k^{\alpha-1} \\
& \leq 2\left(1-\frac{\sqrt{2}}{2}\right)^{-1} q^{-\alpha} \leq 2\left(1-\frac{\sqrt{2}}{2}\right)^{-1} k^{-\alpha} \\
& \leq 2\left(1-\frac{\sqrt{2}}{2}\right)^{-1} k^{-\frac{1}{2}} .
\end{aligned}
$$

Equivalently,

$$
\frac{\psi_{\alpha}\left(\frac{q}{k-1}\right)}{\psi_{\alpha}\left(\frac{q}{k}\right)} \leq 1+2\left(1-\frac{\sqrt{2}}{2}\right)^{-1} k^{-\frac{1}{2}} .
$$

So, assume that $q \leq k$. Applying the Mean Value Theorem once more, we get

$$
\psi_{\alpha}\left(\frac{q}{k}\right)=\alpha \frac{q}{k}(1+\gamma)^{-(1+\alpha)} \geq \alpha \frac{q}{k} 2^{-(1+\alpha)} \geq \frac{1}{2} 2^{-2} a \frac{q}{k}=\frac{1}{8} \frac{q}{k}
$$


CONTINUITY OF THE HAUSDORFF MEASURE OF CONTINUED FRACTIONSANDCOUNTABLE ALPHABET ITERATED F for some $\gamma \in[0, q / k] \subset[0,1]$. Therefore, using also (3.11), we get

$$
\frac{\psi_{\alpha}\left(\frac{q}{k-1}\right)-\psi_{\alpha}\left(\frac{q}{k}\right)}{\psi_{\alpha}\left(\frac{q}{k}\right)} \leq 16 \frac{q}{k^{2}}\left(1+\frac{q}{k}\right)^{-(1+\alpha)} \frac{k}{q} \leq \frac{16}{k} .
$$

Equivalently,

$$
\frac{\psi_{\alpha}\left(\frac{q}{k-1}\right)}{\psi_{\alpha}\left(\frac{q}{k}\right)} \leq 1+\frac{16}{k}
$$

Along with 3.12 this shows that 3.10 holds, and the proof is complete.

Now for every $k \geq 2$ let

$$
\mathcal{M}_{k}^{+}=\left\{\left[\frac{1}{l+q}, \frac{1}{l}\right]: k \leq l, q \geq 1\right\} .
$$

We shall prove the following.

Lemma 3.24. The sequence $\left(\mathcal{M}_{k}^{+}\right)_{k=2}^{\infty}$ is extremal.

Proof. Since $m_{n}\left(\left[0, \frac{1}{n+1}\right]\right)=0$ we are to show that

$$
\varliminf_{\substack{k \rightarrow \infty \\ n \rightarrow \infty}} \inf \left\{\frac{\left(\frac{1}{l}-\frac{1}{l+q}\right)^{h_{n}}}{m_{n}\left(\left[\frac{1}{l+q}, \frac{1}{l}\right]\right)}: q \geq 1, k \leq l \leq l+q \leq n+1\right\} \geq 1 .
$$

Equivalently,

$$
\varlimsup_{\substack{k \rightarrow \infty \\ n \rightarrow \infty}} \sup \left\{\frac{m_{n}\left(\left[\frac{1}{l+q}, \frac{1}{l}\right]\right)}{\left(\frac{1}{l}-\frac{1}{l+q}\right)^{h_{n}}}: q \geq 1, k \leq l \leq l+q \leq n+1\right\} \leq 1 .
$$

But

$$
\begin{aligned}
m_{n}\left(\left[\frac{1}{l+q}, \frac{1}{l}\right]\right) & =\sum_{j=l}^{l+q-1} m_{n}\left(\left[\frac{1}{j+1}, \frac{1}{j}\right]\right) \leq \sum_{j=l}^{l+q-1} \frac{1}{j^{2 h_{n}}} \\
& \leq \int_{l-1}^{l+q-1} x^{-2 h_{n}} d x \\
& =\frac{1}{2 h_{n}-1}\left((l-1)^{1-2 h_{n}}-(l-1+q)^{1-2 h_{n}}\right) .
\end{aligned}
$$

So, it is enough to show that

$$
\varlimsup_{\substack{k \rightarrow \infty \\ n \rightarrow \infty}} \sup \left\{\frac{(l-1)^{1-2 h_{n}}-(l-1+q)^{1-2 h_{n}}}{\left(\frac{1}{l}-\frac{1}{l+q}\right)^{h_{n}}}: q \geq 1, k \leq l \leq l+q \leq n+1\right\} \leq 1 .
$$


Since $1 / 2 \leq 2 h_{n}-1 \leq 1$ for all $n \geq 2$ large enough, by virtue of Lemma 3.23 , it thus suffices to show that

$$
\varlimsup_{\substack{k \rightarrow \infty \\ n \rightarrow \infty}} \sup \left\{\frac{l^{1-2 h_{n}}-(l+q)^{1-2 h_{n}}}{\left(\frac{1}{l}-\frac{1}{l+q}\right)^{h_{n}}}: q \geq 1, k \leq l \leq l+q \leq n+1\right\} \leq 1 .
$$

We have

$$
\begin{aligned}
\frac{l^{1-2 h_{n}}-(l+q)^{1-2 h_{n}}}{\left(\frac{1}{l}-\frac{1}{l+q}\right)^{h_{n}}} & =\frac{(l+q)^{1-2 h_{n}}\left(\left(\frac{l}{l+q}\right)^{1-2 h_{n}}-1\right)}{(l+q)^{-h_{n}}\left(\frac{l}{l+q}-1\right)^{h_{n}}} \\
& =(l+q)^{1-h_{n}} \frac{\left(\frac{l+q}{l}\right)^{2 h_{n}-1}-1}{\left(\frac{l+q}{l}-1\right)^{h_{n}}} \\
& \leq(l+q)^{1-h_{n}} \frac{\frac{l+q}{l}-1}{\left(\frac{l+q}{l}-1\right)^{h_{n}}}=(l+q)^{1-h_{n}}\left(\frac{l+q}{l}-1\right)^{1-h_{n}} \\
& =(l+q)^{1-h_{n}}\left(\frac{q}{l}\right)^{1-h_{n}} \leq(q(l+q))^{1-h_{n}} \\
& \leq(l+q)^{2\left(1-h_{n}\right)} \leq(n+1)^{2\left(1-h_{n}\right)} \\
& \leq\left((n+1)^{\frac{1}{n}}\right)^{\frac{14}{\pi^{2}}},
\end{aligned}
$$

where the last inequality holds for all $n \geq 2$ large enough due to (3.4). Since $\lim _{n \rightarrow \infty}(n+1)^{\frac{1}{n}}=1$, formula 3.13$)$ is established and the proof is complete.

For every $k \geq 1$ let $\mathcal{N}_{k}^{+}$be the family of all closed intervals contained in $[0,1 / k]$. We shall prove the following.

Lemma 3.25. The sequence $\left(\mathcal{N}_{k}^{+}\right)_{1}^{\infty}$ is extremal.

Proof. It follows from Lemmas 3.21 and 3.22 along with Lemma 3.15 that the sequence $\left(\mathcal{S}_{k}^{-} \cup \mathcal{S}_{k}^{+}\right)_{1}^{\infty}$ is extremal. Hence, by virtue of Lemma 3.14 the sequence $\left(\bigcup_{l=k}^{\infty}\left(\mathcal{S}_{k}^{-} \cup \mathcal{S}_{k}^{+}\right)\right)_{1}^{\infty}$ is extremal. Since $\mathcal{N}_{k}^{+} \subset \mathcal{S}_{k}^{-} \oplus \mathcal{M}_{k}^{+} \oplus \mathcal{S}_{k}^{+}$, the proof is therefore concluded by invoking Lemma 3.24, Lemma 3.18, and Lemma 3.13 .

Let $\mathcal{F}$ be the family of all closed intervals in $[0,1]$. We shall prove the following.

Proposition 3.26. The family $\mathcal{F}$ is extremal. 
CONTINUITY OF THE HAUSDORFF MEASURE OF CONTINUED FRACTIONSANDCOUNTABLE ALPHABET ITERATED F

Proof. Proceeding by contradiction suppose that the family $\mathcal{F}$ is not extremal. This means that there are $\eta \in(0,1)$ and two sequences, $\left(n_{j}\right)_{1}^{\infty}$ of strictly increasing positive integers, and $\left(F_{j}\right)_{1}^{\infty}$ of closed intervals in $[0,1]$ such that

$$
\frac{\left|F_{j}\right|^{h_{n_{j}}}}{m_{n_{j}}\left(F_{j}\right)}<\eta
$$

for all $j \geq 1$. For every $j \geq 1$ let $\omega^{(j)} \in \mathbb{N}^{*}$ be the longest word such that

$$
F_{j} \subset g_{\omega(j)}([0,1]) .
$$

Denote

$$
l_{j}:=\left|\omega^{(j)}\right|
$$

Fix

$$
\eta<\xi<1
$$

By Lemma 3.25 and Lemma 3.3 there exists an integer $N \geq 2$ so large that if

$$
G^{k}\left(F_{j}\right) \subset[0,1 / N]
$$

for some $0 \leq k \leq l_{j}$, then, with the help of Lemma 3.2 (a),

$$
\kappa\left(\left.G^{k}\right|_{F_{j}}\right)<\xi / \eta
$$

and

$$
\frac{\left|G^{k}\left(F_{j}\right)\right|^{h_{n_{j}}}}{m_{n_{j}}\left(G^{k}\left(F_{j}\right)\right)}>\xi
$$

Using these two latter inequalities alone and invoking (3.14), we get

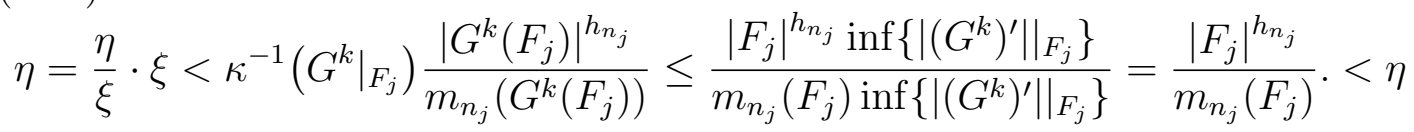

This contradiction shows that

$$
G^{k}\left(F_{j}\right) \cap[1 / N, 1] \neq \emptyset
$$

for all $j \geq 1$ and all $0 \leq k \leq l_{j}$. By the definition of $\omega^{(j)}$ (particularly its length), we then also have

$$
G^{l_{j}}\left(F_{j}\right) \cap\left\{\frac{1}{N}, \frac{1}{N-1}, \frac{1}{N-2}, \ldots, \frac{1}{2}, 1\right\} \neq \emptyset .
$$

and

$$
G^{k}\left(F_{j}\right) \subset\left[\frac{1}{i_{j, k}+1}, \frac{1}{i_{j, k}}\right]
$$

for all $0 \leq k \leq l_{j}-1$ and some $i_{j, k} \in\{1,2, \ldots, N\}$. By Lemma 3.3 there exists $s \in(0,1]$ so small that

$$
\kappa\left(\left.g_{\omega}\right|_{\Delta}\right)<\xi / \eta
$$


for all $\omega \in \mathbb{N}^{*}$ and all intervals $\Delta \subset[0,1]$ with $|\Delta| \leq s$. Now we shall show that

$$
\varliminf_{j \rightarrow \infty} \max \left\{\left|G^{q}\left(F_{j}\right)\right|: 0 \leq q \leq l_{j}\right\}=0 .
$$

Indeed, assume on the contrary that this lower limit is positive. This means that there exist $\theta>0$ and an integer $P_{1} \geq 1$ such that

$$
\max \left\{\left|G^{q}\left(F_{j}\right)\right|: 0 \leq q \leq l_{j}\right\}>\theta
$$

for all $j \geq P_{1}$. This in turn means that for every $j \geq P_{1}$ there exists $q_{j} \in\left\{0, \ldots, l_{j}\right\}$ such that

$$
\left|G^{q_{j}}\left(F_{j}\right)\right|>\min \{\theta, s\} .
$$

We fix the least $q_{j} \in\left\{0, \ldots, l_{j}\right\}$ with this property. By Lemma 3.8 and by (3.14),

$$
\lim _{j \rightarrow \infty}\left|F_{j}\right|=0
$$

Therefore there exists $P_{2} \geq P_{1}$ such that $q_{j} \geq 1$ for all $j \geq P_{2}$. It then follows from, (3.19), and the definition of $q_{j}$ (the least one) that

$$
\gamma \leq\left|G^{q_{j}-1}\left(F_{j}\right)\right| \leq \min \{\theta, s\} \leq s
$$

for all $j \geq P_{2}$ and some $\gamma>0$. Hence, in view of Lemma 3.8 again and of (3.22) there exists $P_{3} \geq P_{2}$ such that

$$
\frac{\left|G^{q_{j}-1}\left(F_{j}\right)\right|^{h_{n_{j}}}}{m_{n_{j}}\left(G^{q_{j}-1}\left(F_{j}\right)\right)}>\xi
$$

for all $j \geq P_{3}$. Making use of this formula, the far-most right-hand side of (3.23), and 3.20 , the calculation of 3.17 ) (with $k=q_{j}-1$ ) goes through to yield $\eta>\eta$. This contradiction finishes the proof of 3.21 .

Now, because of (3.18), Lemma 3.20 implies that

$$
\varliminf_{j \rightarrow \infty} \frac{\left|G^{l_{j}}\left(F_{j}\right)\right|^{h_{n_{j}}}}{m_{n_{j}}\left(G^{l_{j}}\left(F_{j}\right)\right)} \geq 1 .
$$

So there exists $P_{4} \geq 2$ so large that

$$
\frac{\left|G^{l_{j}}\left(F_{j}\right)\right|^{h_{n_{j}}}}{m_{n_{j}}\left(G^{l_{j}}\left(F_{j}\right)\right)}>\xi
$$

for all $j \geq P_{4}$. On the other hand 3.21 entails

$$
\varliminf_{j \rightarrow \infty}\left|G^{l_{j}}\left(F_{j}\right)\right|=0
$$

Hence, there exists $j \geq P_{4}$ such that

$$
\left|G^{l_{j}}\left(F_{j}\right)\right| \leq s
$$

Having this, (3.24), and (3.20), the calculation of (3.17), performed the third time (now with $k=l_{j}$ ), yields again $\eta>\eta$. This contradiction finishes the proof. 
As an immediate consequence of this proposition and Corollary 2.4, we get the following.

$$
\varliminf_{n \rightarrow \infty} \mathrm{H}_{h_{n}}\left(J_{n}(\mathcal{G})\right) \geq 1 .
$$

In order to complete the proof Theorem 3.1, we also need the following, much easier to prove, formula.

$$
\varlimsup_{n \rightarrow \infty} \mathrm{H}_{h_{n}}\left(J_{n}(\mathcal{G})\right) \leq 1 .
$$

Indeed, let $\sigma: \mathbb{N}^{\mathbb{N}} \rightarrow \mathbb{N}^{\mathbb{N}}$ be the shift map, i.e. $\sigma(\omega)$ is uniquely defined be declaring that for every $n \in \mathbb{N}$ its $n$th coordinate is equal to $\omega_{n+1}$. We denote by $\pi(\omega)$ the unique element of $[0,1]$ whose continued fraction representation is equal to $\omega$. So, we have defined an injective Borel map $\pi: \mathbb{N}^{\mathbb{N}} \rightarrow[0,1]$. Its restriction to $\mathbb{N}_{n}^{\mathbb{N}}$ is then a Borel bijection onto $J_{n}(\mathcal{G})$. Denote by $\tilde{m}_{n}$ the image of $m_{n}$ under the inverse of $\left.\pi\right|_{\mathbb{N}_{n}^{\mathbb{N}}}$. It is known from [MU2] that there exists $\tilde{\mu}_{n}$, a unique Borel probability measure $\sigma$-invariant measure on $\mathbb{N}_{n}^{\mathbb{N}}$, absolutely continuous with respect to $\tilde{m}_{n}$. In addition, $\tilde{\mu}_{n}$ is ergodic with respect to $\sigma: \mathbb{N}_{n}^{\mathbb{N}} \rightarrow \mathbb{N}_{n}^{\mathbb{N}}$ and equivalent to $\tilde{m}_{n}$. Now for every $\omega \in\{1,2, \ldots, n\}^{\mathbb{N}}$ let

$$
Z(\omega):=\left\{j \geq 1: \omega_{j}=\left(\sigma^{j-1}(\omega)\right)_{1}=n\right\} .
$$

Because of Birkhoff's Ergodic Theorem, ergodicity of the measure $\tilde{\mu}_{n}$, and positivity of $\tilde{\mu}_{n}\left(\left[\frac{1}{n+1}, \frac{1}{n}\right]\right)$, there exists a Borel set $\Gamma_{n} \subset\{1,2, \ldots, n\}^{\mathbb{N}}$ with $\tilde{\mu}_{n}\left(\Gamma_{n}\right)=1$ (equivalently $\tilde{m}_{n}\left(\Gamma_{n}\right)=1$ ) such that for every $\omega \in \Gamma_{n}$ the set

$$
Z_{n}(\omega):=\left\{j \geq 1: \omega_{j}=\left(\sigma^{j-1}(\omega)\right)_{1}=n\right\}
$$

is infinite. Now fix $\varepsilon>0$. By virtue of Lemma 3.6 there exists $N_{\varepsilon} \geq 1$ such

$$
\kappa\left(g_{\left.\omega\right|_{j}}\right) \leq 1+\varepsilon
$$

for $n \geq N_{\varepsilon}$, all $\omega \in \Gamma_{n}$, and all $j \in Z_{n}(\omega)$. But then, using Lemma 3.2(d), we get

$$
\frac{\operatorname{diam}^{h_{n}}\left(g_{\left.\omega\right|_{j}}([0,1])\right)}{m_{n}\left(g_{\left.\omega\right|_{j}}([0,1])\right)} \leq \frac{\kappa^{h_{n}}\left(g_{\left.\omega\right|_{j}}\right) \inf ^{h_{n}}\left\{\left|g^{\prime}\right|_{\left.\omega\right|_{j}} \mid\right\}}{\inf ^{h_{n}}\left\{\left|g^{\prime}\right|_{\left.\omega\right|_{j}} \mid\right\}}=\kappa^{h_{n}}\left(g_{\left.\omega\right|_{j}}\right) \leq \kappa\left(g_{\left.\omega\right|_{j}}\right) \leq 1+\varepsilon .
$$

Along with (3.5) this implies that

$$
\lim _{r \rightarrow 0} \inf \left\{\frac{\operatorname{diam}^{h_{n}}(F)}{m_{n}(F)}: \omega \in \Gamma_{n}, \pi(\omega) \in F, \operatorname{diam}(F) \leq r\right\} \leq 1+\varepsilon .
$$

As $m_{n}\left(\pi\left(\Gamma_{n}\right)\right) \geq \tilde{m}_{n}\left(\Gamma_{n}\right)=1$, by Corollary 2.4, this gives that $\mathrm{H}_{h_{n}}\left(J_{n}(\mathcal{G})\right) \leq 1+\varepsilon$ for all $n \geq N_{\varepsilon}$. The formula $(3.26)$ is proved.

Now, formulas $(3.25)$ and $(3.26)$ taken together, prove Theorem 3.1. 


\section{Short Preliminaries on Conformal Iterated Function Systems}

Let $(X, \rho)$ be a compact metric space. Let $E$ be a countable set, either finite or infinite, called in the sequel an alphabet. Fix a number $s \in(0,1)$. Suppose that for every $e \in E$ there is given an injective contraction $\phi_{i}: X \rightarrow X$. with a Lipschitz constant $\leq s$. The collection

$$
S=\left\{\phi_{e}: X \rightarrow X\right\}_{e \in E}
$$

is called an iterated function system; briefly an IFS. Our main object of interest is the limit set of the system $S$. We will now define it. For each $\omega \in E^{*}$, say $\omega \in E^{n}$, we consider the map coded by $\omega$ :

$$
\phi_{\omega}:=\phi_{\omega_{1}} \circ \cdots \circ \phi_{\omega_{n}}: X \rightarrow X .
$$

For every $\omega \in E^{\mathbb{N}}$, the sets $\left\{\phi_{\left.\omega\right|_{n}}(X)\right\}_{n \geq 1}$ form a descending sequence of nonempty compact sets and therefore $\bigcap_{n \geq 1} \phi_{\left.\omega\right|_{n}}(X) \neq \emptyset$. Since for every $n \geq 1$,

$$
\operatorname{diam}\left(\phi_{\left.\omega\right|_{n}}(X)\right) \leq s^{n} \operatorname{diam}(X),
$$

we conclude that the intersection

$$
\bigcap_{n \geq 1} \phi_{\left.\omega\right|_{n}}(X)
$$

is a singleton and we denote its only element by $\pi(\omega)$. In this way we have defined the coding map $\pi$ the coding map from the coding space to the limit set $\pi$ :

$$
\pi: E^{\mathbb{N}} \rightarrow X
$$

from $E^{\mathbb{N}}$ to $X$. The set

$$
J:=J_{S}=\pi\left(E^{\mathbb{N}}\right)
$$

will be called the limit set of the IFS $S$. An IFS $S$ is called conformal if the following conditions are satisfied.

(a) $X$ is a compact connected subset of a Euclidean space $\mathbb{R}^{d}$ and $X=\overline{\operatorname{Int}(X)}$.

(b) (Cone Condition) There exist $\alpha, l>0$ such that for every $x \in \partial X \subset$ $\mathbb{R}^{d}$ there exists an open cone $\operatorname{Con}(x, u, \alpha) \subset \operatorname{Int}(X)$ with vertex $x$, the symmetry axis determined by the vector $u \in \mathbb{R}^{d}$ of length $l$ and a central angle of Lebesgue measure $\alpha$. Here $\operatorname{Con}(x, u, \alpha, l)=\{y: 0<(y-x, u) \leq$ $\cos \alpha\|y-x\| \leq l\}$.

(c) (Open set Condition; OSC). For all $a, b \in E, a \neq b$, it holds

$$
\phi_{a}\left(\operatorname{Int}(X) \cap \phi_{b}(\operatorname{Int}(X)=\emptyset .\right.
$$

(d) There exists an open connected set $\mathbb{R}^{d} \supset W \supset X$ such that for every $e \in E$, the map $\phi_{e}$ extends to a $C^{1}$ conformal diffeomorphism of $W$ into $W$. 
(e) (Bounded Distortion Property) There exist a constant $K \geq 1$ and $\alpha \in$ $(0,1]$ such that

$$
\left|\frac{\left|\phi_{\omega}^{\prime}(y)\right|}{\left|\phi_{\omega}^{\prime}(x)\right|}-1\right| \leq K|| y-x \|^{\alpha}
$$

and

$$
K^{-1} \leq \frac{\left|\phi_{\omega}^{\prime}(y)\right|}{\left|\phi_{\omega}^{\prime}(x)\right|} \leq K
$$

for every $\omega \in E^{*}$ and every pair of points $x, y \in X$.

Remark 4.1. Observe that the Cone condition is automatically satisfied if $d=1$. Also, (see [MU2]) the Bounded Distortion Property is satisfied if either $d \geq 2$, or else if $d=1$ and the alphabet $E$ is finite. It is also trivially satisfied whenever the system $S$ consists of similarities only. Finally, decreasing a constant $K$ if necessary, the latter property in (e) follows from the former.

For every $t \geq 0$ define

$$
\mathrm{P}(t):=\lim _{n \rightarrow \infty} \frac{1}{n} \log \sum_{\omega \in E^{n}}\left\|\phi_{\omega}^{\prime}\right\|_{\infty}^{t} .
$$

The limit exists indeed since the corresponding sequence is subadditive. It is called the topological pressure of $t$. If the system $S$ consists of similarities only, then the pressure is easy to calculate. We have,

$$
\mathrm{P}(t)=\log \sum_{e \in E}\left|\phi_{e}^{\prime}\right|^{t} .
$$

The following formula, called Bowen's formula, was proved in [MU2].

$$
\operatorname{HD}\left(J_{S}\right)=\inf \{t \geq 0: \mathrm{P}(t) \leq 0\}=\sup \left\{\operatorname{HD}\left(J_{F}\right): F \subset E \text { is finite }\right\} .
$$

$J_{F}$ in here is the limit set of the iterated function system $\left\{\phi_{e}: X \rightarrow X\right\}_{e \in F}$. If all elements of the system $S$ are similarities, then this formula simplifies to read the following.

$$
\operatorname{HD}\left(J_{S}\right)=\inf \left\{t \geq 0: \sum_{e \in E}\left|\phi_{e}^{\prime}\right|^{t} \leq 1\right\}=\sup \left\{\operatorname{HD}\left(J_{F}\right): F \subset E \text { is finite }\right\} .
$$

Remark 4.2. If there exists a parameter $t \geq 0$ such that $\mathrm{P}(t)=0$, meaning that

$$
\sum_{e \in E}\left|\phi_{e}^{\prime}\right|^{t}=1
$$

in case of similarities, then this $t$ is unique and is equal to $\operatorname{HD}\left(J_{S}\right)$. The system $S$ is then called regular. All finite alphabet systems are obviously regular. 


\section{HausdorfF Measures for Similarity IFSs}

In this section we prove a considerably simplified formulas for the numerical value of the Hausdorff measure of the limit set of a conformal (either finite or infinite) IFS consisting of similarities only. It will be extensively used in the next section, where a counterexample for continuity of Hausdorff measure is constructed.

Theorem 5.1. If $\mathcal{S}=\left\{\phi_{e}: X \rightarrow X\right\}_{e \in E}$ is a conformal (either finite or infinite) IFS consisting of similarities only, and $\mathrm{H}_{h}\left(J_{\mathcal{S}}\right)>0$, then

$$
\mathrm{H}_{h}\left(J_{\mathcal{S}}\right)=\inf \left\{\frac{\operatorname{diam}^{h}(F)}{\mathrm{H}_{h}^{1}(F)}: F \subset X, \bar{F}=F\right\} .
$$

Proof. Since

$\inf \left\{\frac{\operatorname{diam}^{h}(F)}{\mathrm{H}_{h}^{1}(F)}: F \subset X, \bar{F}\right\} \leq \liminf _{r \rightarrow 0}\left\{\frac{\operatorname{diam}^{h}(F)}{\mathrm{H}_{h}^{1}(F)}: x \in F, \bar{F}=F, \operatorname{diam}(F) \leq r\right\}$

for every $x \in X$, as an immediate consequence of Theorem 2.2, we get that

$$
\inf \left\{\frac{\operatorname{diam}^{h}(F)}{\mathrm{H}_{h}^{1}(F)}: F \subset X, \bar{F}=F\right\} \leq \mathrm{H}_{h}\left(J_{\mathcal{S}}\right)
$$

In order to prove the opposite inequality fix $\varepsilon>0$. Denote the left-hand side of (5.1) by $L$. Fix a closed subset $F$ of $X$ such that

$$
\frac{\operatorname{diam}^{h}(F)}{\mathrm{H}_{h}^{1}(F)} \leq L+\varepsilon
$$

and

$$
\mathrm{H}_{h}^{1}(F)>0
$$

Given $\omega \in E^{\mathbb{N}}$ let

$$
Z(\omega):=\left\{j \geq 0: \sigma^{j}(\omega) \in \pi^{-1}(F)\right\} .
$$

Since $\tilde{\mu}_{h}\left(\pi^{-1}(F)\right)=\mu_{h}(F)>0$, it follows from Birkhoff's Ergodic Theorem (and ergodicity of $\tilde{\mu}_{h}$ with respect to the the shift map $\sigma: E^{\mathbb{N}} \rightarrow E^{\mathbb{N}}$ that $\tilde{\mu}_{h}(\Gamma)=1$, where

$$
\Gamma:=\left\{\omega \in^{\mathbb{N}}: Z(\omega) \text { is infinite }\right\} .
$$

Let $\omega \in \Gamma$ and $j \in Z(\omega)$. Then

$$
\pi(\omega)=\phi_{\left.\omega\right|_{j}}\left(\pi\left(\sigma^{j}(\omega)\right) \in \phi_{\left.\omega\right|_{j}}(F)\right.
$$

and, using (5.2),

$$
\frac{\operatorname{diam}^{h}\left(\phi_{\left.\omega\right|_{j}}(F)\right)}{\mathrm{H}_{h}^{1}\left(\phi_{\left.\omega\right|_{j}}(F)\right)}=\frac{\left|\phi_{\left.\omega\right|_{j}}^{\prime}\right|^{h} \operatorname{diam}^{h}(F)}{\left|\phi_{\left.\omega\right|_{j}}^{\prime}\right|{ }^{h} \mathrm{H}_{h}^{1}(F)}=\frac{\operatorname{diam}^{h}(F)}{\mathrm{H}_{h}^{1}(F)} \leq L+\varepsilon .
$$


CONTINUITY OF THE HAUSDORFF MEASURE OF CONTINUED FRACTIONSANDCOUNTABLE ALPHABET ITERATED F

Since $Z(\omega)$ is unbounded and since $\mathrm{H}_{h}^{1}(\pi(\Gamma)) \geq \widetilde{\mathrm{H}}_{h}^{1}(\Gamma) \geq 1$, the last two formulas, in conjunction with Theorem $2.2(\pi(\omega)$ plays the role of $x$ appearing there), imply that $\mathrm{H}_{h}\left(J_{\mathcal{S}}\right) \leq L+\varepsilon$. Letting $\varepsilon \rightarrow 0^{+}$, this yields $\mathrm{H}_{h}\left(J_{\mathcal{S}}\right) \leq L$. Along with (5.1) this completes the proof.

Since in all Euclidean metric spaces the diameter of the closed convex hull of every set $A$ is the same as the diameter of $A$, as an immediate consequence of this theorem, we get the following.

Corollary 5.2. If $\mathcal{S}=\left\{\phi_{e}: X \rightarrow X\right\}_{e \in E}$ is a conformal (either finite or infinite), IFS consisting of similarities only, $\mathrm{H}_{h}\left(J_{\mathcal{S}}\right)>0$, and $X$ is a convex set, then

$$
\mathrm{H}_{h}\left(J_{\mathcal{S}}\right)=\inf \left\{\frac{\operatorname{diam}^{h}(F)}{\mathrm{H}_{h}^{1}(F)}: F \subset X \text { is closed and convex }\right\} \text {. }
$$

Being even more specific, we get the following consequence.

Corollary 5.3. If $\mathcal{S}=\left\{\phi_{e}: X \rightarrow X\right\}_{e \in E}$ is a conformal (either finite or infinite) IFS consisting of similarities only, $\mathrm{H}_{h}\left(J_{\mathcal{S}}\right)>0$, and $X$ is a closed bounded subinterval of $\mathbb{R}$, then

$$
\mathrm{H}_{h}(X)=\inf \left\{\frac{\operatorname{diam}^{h}(F)}{\mathrm{H}_{h}^{1}(F)}: F \subset X \text { is a closed interval }\right\} .
$$

\section{One Dimensional Linear Counterexample}

One of the major technical issues in the proof of Theorem 3.1 was to have the derivative distortion so close to one as desired. As the counterexample, for continuity of the Hausdorff measure, described below shows, this was not the only problem.

Example 6.1. We will construct by induction an infinite iterated function system $\mathcal{S}=\left\{\phi_{n}: X \rightarrow X\right\}_{n \in \mathbb{N}}$ with the following properties.

(a) $X=[0,1]$.

(b) $\mathcal{S}$ consists of decreasing similarities only.

(c)

$$
\bigcup_{n=0}^{\infty} \phi_{n}([0,1])=(0,1]
$$


and, consequently,

$$
J_{\mathcal{S}}=[0,1] \backslash \bigcup_{\omega \in \mathbb{N}^{*}} \phi_{\omega}(0) .
$$

(d)

$$
\varliminf_{n \rightarrow \infty} \mathrm{H}_{h_{n}}\left(J_{n}\right)=0<1=\mathrm{H}_{1}\left(J_{\mathcal{S}}\right),
$$

where $J_{n}=J_{\mathcal{S}_{n}}$ is the limit set of the iterated function system $\mathcal{S}_{n}:=$ $\left\{\phi_{0}, \phi_{1}, \ldots, \phi_{n}\right\}$, and $h_{n}:=\operatorname{HD}\left(J_{n}\right)$.

We define $I_{1}:=\{1\}$ and $\phi_{1}:[0,1] \rightarrow[0,1]$ to be the unique linear (decreasing) map such that

$$
\phi_{1}(0)=1 \text { and } \phi_{1}(1)=1 / 2 .
$$

Proceeding inductively suppose that $n \geq 2$ and $I_{n-1}$, an initial finite block of $\mathbb{N}$ has been defined along with the linear decreasing maps $\phi_{i}:[0,1] \rightarrow[0,1]$, $i \in I_{n-1}$, satisfying the following properties

(e)

$$
\left[\frac{1}{n-1}, 1\right] \subset \bigcup_{i \in I_{n-1}} \phi_{i}([0,1]) \subset\left(\frac{1}{n}, 1\right]
$$

(f) $\phi_{i}(0)=\phi_{i-1}(1)$ for all $i \in I_{n-1} \backslash\{1\}$.

Let $N_{n-1}$ be the largest number in $I_{n-1}$. Fix a point $\xi_{n} \in\left(\frac{1}{n+1}, \frac{1}{n}\right)$, for example $\frac{1}{2}\left(\frac{1}{n+1}+\frac{1}{n}\right)$. Let $\phi_{N_{n-1}+1}:[0,1] \rightarrow[0,1]$ be the unique linear (decreasing) map such that

(g) $\phi_{N_{n-1}+1}(1)=\xi_{n}$ and $\phi_{N_{n-1}+1}(0):=\phi_{N_{n-1}}(1)$ is the left-hand endpoint of $\bigcup_{i \in I_{n-1}} \phi_{i}([0,1])$.

Let

$$
\mathcal{R}_{n}^{*}:=\left\{\phi_{j}: 1 \leq j \leq N_{n-1}+1\right\}
$$

and let

$$
s_{n}^{*}:=\operatorname{HD}\left(J_{\mathcal{R}_{n}^{*}}\right)
$$

Fix

$$
\gamma_{n} \in\left(s_{n}^{*}, 1\right) .
$$

Take an integer $k_{n} \geq 1$ so large that

$$
\left(1-\gamma_{n}\right) \log k_{n} \geq \log n .
$$

Since, by Remark 4.2 ,

$$
\sum_{i=1}^{N_{n-1}+1}\left|\phi_{i}^{\prime}\right|^{s_{n}^{*}}=1
$$


CONTINUITY OF THE HAUSDORFF MEASURE OF CONTINUED FRACTIONSANDCOUNTABLE ALPHABET ITERATED F

there exists $a_{n} \in(0,1)$ so small that

$$
\sum_{i=1}^{N_{n-1}+1}\left|\phi_{i}^{\prime}\right|^{\gamma_{n}}+k_{n} a_{n}^{\gamma_{n}}=\sum_{i \in I_{n-1} \cup\left\{N_{n-1}+1\right\}}\left|\phi_{i}^{\prime}\right|^{\gamma_{n}}+k_{n} a_{n}^{\gamma_{n}}<1 .
$$

Let

$$
I_{n}^{*}:=\left\{N_{n-1}+2, N_{n-1}+3, \ldots, N_{n-1}+k_{n}+2\right\}
$$

and let

$$
I_{n}:=I_{n-1} \cup\left\{N_{n-1}+1\right\} \cup I_{n}^{*}=\left\{1,2 \ldots, N_{n-1}+k_{n}+2\right\} .
$$

Now, for every $N_{n-1}+2 \leq i \leq N_{n-1}+k_{n}+2$, let $\phi_{i}:[0,1] \rightarrow[0,1]$ be a linear (decreasing) map with the following properties:

(h) The scaling factor of $\phi_{i}$ is equal to $a_{n}$ for all $i=N_{n-1}+2, \ldots, N_{n-1}+k_{n}+2$,

(i) $\phi_{N_{n-1}+2}(0)=\phi_{N_{n-1}+1}(1)$,

(j) $\phi_{i+1}(0)=\phi_{i}(1)$ for all $i=N_{n-1}+2, \ldots, N_{n-1}+k_{n}+1$.

We set

$$
\mathcal{R}_{n}:=\left\{\phi_{i}\right\}_{i \in I_{n}}
$$

Formula 6.2 implies that

$$
s_{n}:=\operatorname{HD}\left(J_{\mathcal{R}_{n}}\right)<\gamma_{n}
$$

Now let

$$
\Delta_{n}:=\bigcup_{i \in I_{n}} \phi_{i}([0,1])
$$

By our construction $\Delta_{n} \subset(0,1]$ is a closed interval and we have

$$
\frac{\operatorname{diam}^{s_{n}}\left(\Delta_{n}\right)}{\hat{m}_{n}\left(\Delta_{n}\right)}=\frac{\left(k_{n} a_{n}\right)^{s_{n}}}{k_{n} a_{n}^{s_{n}}}=k_{n}^{s_{n}-1}
$$

where $\hat{m}_{n}$ is the only $s_{n}$-conformal measure for the system $\mathcal{R}_{n}$. By 6.3) and (6.1), we get

$$
\log \left(k_{n}^{s_{n}-1}\right)=\left(s_{n}-1\right) \log k_{n}<\left(\gamma_{n}-1\right) \log k_{n}<-\log n=\log (1 / n),
$$

and therefore,

$$
\frac{\operatorname{diam}^{s_{n}}\left(\Delta_{n}\right)}{\hat{m}_{n}\left(\Delta_{n}\right)}<\frac{1}{n}
$$

By construction, $\left(I_{n}\right)_{1}^{\infty}$ is an ascending sequence of initial blocks of $\mathbb{N}, \bigcup_{n=1}^{\infty} I_{n}=$ $\mathbb{N},\left.\mathcal{R}_{n+1}\right|_{I_{n}}=\mathcal{R}_{n}$, and we define

$$
\mathcal{S}=\bigcup_{n=1}^{\infty} \mathcal{R}_{n}
$$

The required properties (a) and (b) then trivially hold for the system $\mathcal{S}$. The property $(c)$ holds by virtue of $(\mathrm{e})$, and $(\mathrm{d})$ holds because of 6.2 , which because of Theorem 5.1. implies that $\mathrm{H}\left(J_{\mathcal{R}_{n}}\right)<1 / n$. 


\section{REFERENCES}

[H1] D. Hensley, Continued fraction Cantor sets, Hausdorff dimension, and functional analysis, J. Number Th. (1992), 40, 336-358. 1, 3, 3, 3

[Ma] P. Mattila, Geometry of sets and measures in Euclidean spaces. Fractals and rectifiability. Cambridge Studies in Advanced Mathematics, 44. Cambridge University Press, Cambridge, 1995. 2. 2.1

[MU1] D. Mauldin, M. Urbański, Dimensions and measures in infinite iterated function systems, Proc. London Math. Soc. (1996), 73, 105-154. 1 , 3

[MU2] D. Mauldin, M. Urbański, Graph Directed Markov Systems: Geometry and Dynamics of Limit Sets, Cambridge Univ. Press (2003). 1, 1, 3, 3, 3, 3, 4.1, 4

[Ol] L. Olsen, Hausdorff and packing measure functions of self- similar sets: continuity and measurability, Ergod. Th. \& Dynam. Sys. (2008), 28, 1635-1655. 1. 2

[SUZ] T. Szarek, M. Urbański, and A. Zdunik, Hausdorff Measure for Conformal Dynamical Systems, Discrete \& Continuous Dynam. Sys., Series A., (2013) 33 4647-4692. 1, 2

Department of Mathematics, University of North Texas, Denton, TX 762031430, USA

E-mail address: urbanski@unt.edu

Institute of Mathematics, University of Warsaw, 02-097 WarszaWa, Poland

E-mail address: A.Zdunik@mimuw.edu.pl 